\title{
Does Temperature have a Metric Structure?*
}

\author{
Bradford Skow
}

\begin{abstract}
Is there anything more to temperature than the ordering of things from colder to hotter? Are there also facts, for example, about how much hotter (twice as hot, three times as hot...) one thing is than another? There certainly are-but the only strong justification for this claim comes from statistical mechanics. What we knew about temperature before the advent of statistical mechanics (what we knew about it from thermodynamics) provided only weak reasons to believe it.
\end{abstract}

1. The tea in the mug on my desk is hotter than the air in my office. And the surface of the sun is hotter than the surface of the earth. Is there anything more to temperature than these kinds of facts-facts about how things are ordered with respect to temperature? Is it also true, for example, that the temperature difference between the surface of the sun and the surface of the earth is some definite number of times larger than the temperature difference between the tea and the air? Are there, in general, also facts about the ratios of lengths of temperature intervals? Or, instead, is it meaningless to ask what these ratios are?

If it does make sense to ask then (I will say) temperature has a metric structure. It is easy to see that temperature has a metric structure when one looks at what statistical mechanics says temperature is. But do we need to appeal to statistical mechanics to justify the claim that temperature has a metric structure? Statistical mechanics, I will argue, provides the only strong justification for this claim. What

*Published in Philosophy of Science 78 (2011): 472-489. 
we knew about temperature before the advent of statistical mechanics (especially what we knew about it from thermodynamics) provided at best only weak reasons to think that temperature has a metric structure.

2. The thesis I am defending might seem outrageous at first. One might be tempted to argue against it like this: the temperature of the surface of the sun is close to 5700 Kelvin and the temperature of the surface of the earth averages something like 300 Kelvin. That is a separation of 5400 Kelvin. But the temperatures of the tea and the air are separated by less than 100 Kelvin. So the temperatures of the sun and the earth are separated by an interval at least 34 times larger than the one that separates the temperatures of the tea and the air. Someone who knows nothing about statistical mechanics can arrive at this conclusion. All he needs is a good way to measure temperature on the Kelvin scale (a scale that was established before statistical mechanics was developed).

But the fact that the difference between the numbers the Kelvin scale assigns to the temperatures of the earth and the sun is 34 times larger than the difference between the numbers the Kelvin scale assigns to the tea and the air does not entail that the first temperature difference itself is 34 times greater than the second, or more generally that temperature differences stand in definite ratios. We have to be careful when drawing conclusions about temperatures themselves from features of the scale(s) we use to measure temperature.

When we use real numbers to represent the values of a physical quantity like temperature we are using relations between the numbers to represent relations between the values of the quantity themselves. (Quantities like temperature are determinables; by the value of a quantity I mean a property that is a determinate of that determinable. One and the same value for temperature may be assigned 0 on the Celsius scale, 32 on the Fahrenheit scale, and 273.15 on the Kelvin scale.) But not every relation among the numbers represents a relation among the values. Take, for example, the use of non-negative real numbers to represent masses on the kilogram scale. The number 1 is mathematically special: it is the only number $y$ satisfying $y x=x$ for all $x$. But there is nothing physically special about the mass value assigned the number 1 on the kilogram scale. So it is possible (for all that has been 
said so far) that when we use the Kelvin scale (or any scale) for temperature, the only aspect of the real numbers that does any representational work is the order of the numbers-higher numbers correspond to hotter temperatures. If this possibility is actual then in the Kelvin scale neither the function $f(x, y, z, w)=|x-y| /|z-w|$ that gives the ratio of the distances between two pairs of numbers nor the function $g(x, y)=x / y$ that gives the ratio of two numbers does any representational work.

The other possibility is that one or both of these mathematical functions does do representational work in the scales we use to measure temperature. In this case there are functions defined on the temperature values themselves, functions that give the set of temperature values their structure, that are represented by the functions on numbers..$^{1}$ The question I started with is: are there such structuring functions on temperature values, or not? $?^{2}$

If there are such functions then there is some natural metric structure on temperature values - a function on temperature values represented by one of the functions on numbers $f$ or $g$ defined above lets us speak of the "distance" between two temperature values, as measured with respect to some unit. That is why I use "temperature has a metric structure" to express the claim that there are such functions. Of course, temperature has more structure if there are objective, scale-independent facts about temperature ratios - if there is a structuring function represented by $g$ than it does if there are only objective, scale-independent facts about ratios of magnitudes of temperature intervals (represented by $f$ ). In the first but not the second

\footnotetext{
${ }^{1}$ Perhaps at a more fundamental level the temperature values are given their structure by a distinguished set of relations on temperature values, not a distinguished set of functions from temperature values to numbers. (This is, for example, the view defended by Mundy (1987).) Even so, the distinguished relations pick out a set of distinguished functions, and it is more convenient for my purposes to talk about a distinguished set of functions.

${ }^{2}$ Stevens (1946) introduced the distinction between ordinal scales, interval scales, and ratio scales of measurement (see also Ellis 1966; Krantz et al 1971). On an ordinal scale, only the order relations among numbers represent relations among the values of the quantity being measured; on an interval scale, the function $f$ also does representational work; on a ratio scale, the function $g$ also does representational work. In Stevens' terminology I am asking which of these scale-types is appropriate for measuring temperature.
} 
case there is a natural zero point for temperature. But the differences between these two structures will not play a role in what follows, so I lump them together as kinds of metric structure.

3. One might maintain that there neither are nor could be any physical quantities with metric structures. I shall call this thesis "conventionalism," for the following reason 3 Whenever we set up a scale for measuring some quantity we must establish some conventions. Conventionalists think that setting up a scale requires more conventions than non-conventionalists do. Take, for example, length. Non-conventionalists think that there are scale-independent facts about the ratios of lengths. They take length to be a paradigm case of a quantity with a metric structure. If this is right then we only need to make one conventional choice in order to set up a scale for measuring length. For a faithful scale for measuring length will have the property that the ratios of the numbers assigned to length values are equal to the scale-independent ratios of the corresponding values. So the only freedom we have when setting up a scale (if the non-conventionalist is right) is freedom to choose which length shall be assigned the number 1 . Once we pick which length value $S$ this is (say by legislating that $S$ is the length value instantiated by a certain bar made of platinum-iridium alloy under certain conditions), anything else's length will be $l / S$, the (scale-independent) ratio of that thing's value $l$ for length to $S: 4$

Conventionalists do not think that this procedure succeeds in setting up a scale for measuring length. For they deny that there are scale-independent facts about length ratios. So an utterance of "Let our scale assign to each length value

${ }^{3}$ I also call it "conventionalism" because it resembles claims that conventionalists like Henri Poincaré made. I am not sure whether Poincaré actually accepted this thesis (see for example Poincare 1905, 17-28). Reichenbach (1957, 14-30) also claims to find more conventions than we might originally have thought existed in our measurement practices.

${ }^{4}$ I suspect that this non-conventionalist view of length is also the majority view. It is certainly the view of the International Bureau of Weights and Measures. Its brochure on the international system of units says "The value of a quantity is generally expressed as the product of a number and a unit. The unit is simply a particular example of the quantity concerned which is used as a reference, and the number is the ratio of the value of the quantity to the unit" $(2006,103)$. 
$l$ the number that is the (scale-independent) ratio of $l$ to $S$ " has a false presupposition. Conventionalists think that we need to establish more conventions, besides a conventional choice of standard, in order to assign a unique number to each length value.

Here is the sort of thing a conventionalist might do to set up a scale for measuring length. He starts, as the non-conventionalist does, but selecting a particular thing - a particular metal bar in this case - to be the scale's standard for length. Then he stipulates that a length $l$ shall be assigned a positive integer $n$ iff $n$ copies of the standard, laid end to end, reach exactly from one end to another of anything that instantiates $l$. (There is a natural way to complete this scale so that each length value is assigned some positive real number.) This last stipulation is an additional convention.

Now, the conventionalist and the non-conventionalist who use the scales described above will agree on the lengths of things-as long as there is nothing funny going on. So it is worth emphasizing the differences between their views. The conventionalist will insist that even after a standard has been adopted he was free to adopt an alternative convention for assigning numbers to lengths and still have a scale that faithfully represents the structure of length. The non-conventionalist will deny this; those alternative conventions result in scales that are not faithful. The non-conventionalist will also say that the convention the conventionalist actually adopted makes what is as a matter of contingent fact a reliable procedure for finding out the lengths of things into a procedure that is reliable by definition. (The conventionalist has made it true by definition that metal bars do not change their length when they are moved around.)

Of course one might deny that length has a metric structure without being a conventionalist. Conventionalists believe that no possible quantity has a metric structure. If conventionalism is true then it follows, of course, that temperature in particular does not have a metric structure. So the debate over conventionalism is certainly relevant to the topic of this paper. But arguments for conventionalism are general, all-purpose arguments that apply to any possible quantity. And I am interested in what we should think about the structure of temperature on the assumption that it is at least possible for a quantity to have a metric structure. So I am going 
to assume that conventionalists' all-purpose arguments do not succeed. We need to look at what temperature is and how it works before we can reach any conclusions about its structure.

4. I will begin investigating what we should think about the structure of temperature in ignorance of statistical mechanics by looking (in this section and the next) at the scales we use to measure temperature. As we saw in the last section, the conventions we make when we establish a scale for measuring some quantity make presuppositions about the structure of that quantity. So what do temperature scales presuppose about the structure of temperature?

This discussion is really just a prelude to my discussion of thermodynamics. As we will see, none of the temperature scales we have used presuppose that temperature has a metric structure. But even if one of them did, the fact that it did would by itself not establish much: we would need to know what reason there is to believe that the presupposition is correct. So why am I discussing temperature scales at all? Because what reason there is in thermodynamics to think that temperature has a metric structure comes from the role the Kelvin scale plays in that theory. So it is worth going over how that scale is established and what its presuppositions are. But before discussing the Kelvin scale I will discuss the "empirical" scales the preceded it.

We have already seen what presuppositions are made when we establish a scale for measuring a quantity with a metric structure. It will be useful to have that example in mind when we look at temperature scales. For the way early scales for measuring temperature were set up is very different from the way the scale for measuring length is set up. Those scales for temperature did not presuppose that temperature has a metric structure.

A mercury-based centigrade scale for measuring temperature, for example, is set up as follows. A small amount of mercury is encased in an otherwise evacuated glass bulb from which a very thin cylinder extends. A mark is made on the cylinder indicating the point to which the mercury extends when the bulb is placed in thermal contact with boiling water and allowed to settle into thermal equilibrium. This is the 100 mark. Another mark is made on the cylinder indicating the point to which 
the mercury extends when the bulb is placed in thermal contact with melting ice and allowed to settle into thermal equilibrium. This is the 0 mark. The idea is that a given thermodynamic system's temperature on this scale will be proportional to the ratio of two distances. Let $p$ be the point on the cylinder coincident with the top of the mercury column when the bulb is placed in thermal contact with the system. We decree that that the number measuring the system's temperature on this scale is equal to $100 \frac{d 1}{d 2}$, where $d 1$ is the distance between the 0 mark and $p$ and $d 2$ is the distance between the 0 mark and the 100 mark. (That is its temperature if $p$ is above the 0 mark; its temperature is $-100 \frac{d 1}{d 2}$ if $p$ is below the 0 mark. $)^{5}$

We can see that the presuppositions about temperature made here are different from the presuppositions made about length (by non-conventionalists). The numbers assigned to lengths on the meter scale are defined in part in terms of the (scale-independent) ratios of length values. But no ratios of temperature values, or ratios of lengths of intervals of temperature values, are used to define the numbers assigned to temperatures on this scale.

We might, of course, believe that temperature has a metric structure, and wonder whether the mercury-centigrade scale faithfully represents that structure. (We might wonder, that is, whether the result of applying the function $|x-y| /|z-w|$ to numbers this scale assigns to temperatures is always equal to the scale-independent ratio of the lengths of the corresponding temperature intervals). Many scientists in the late 18 th and early 19 th century did believe and wonder just these things. (Some of them wondered this because they accepted the caloric theory of heat, which (we will see below) says that temperature has a metric structure). For example, Johann Lambert wrote that "One hesitates as to whether the actual degrees of heat are really proportional to the degrees of the expansion" of the substance in the thermometer. Similarly, Joseph Gay-Lussac wrote that "The thermometer as it is today cannot

\footnotetext{
${ }^{5}$ The mercury-based centigrade scale is not the same as (what we currently call) the Celsius scale. Both assign 0 to water's freezing point and 100 to its boiling point (under standard conditions), but they differ over how intermediate numbers are assigned. The first scale assigns intermediate numbers so that (by definition) mercury expands linearly with temperature. Mercury does not expand linearly with temperature on the Celsius scale. By definition, something's temperature in Celsius is just its temperature in Kelvin, minus 273.15.
} 
serve to indicate the exact proportion of heat, as we do not yet know what relation there is between the degrees of the thermometer and the quantity of heat which it is supposed to indicate. We generally believe, it is true, that equal divisions of the tube represent equal quantities of the caloric, but this opinion is not founded on anything positive.' ${ }^{6}$ Still, even if scientists frequently wondered whether temperature scales like the mercury-based centigrade scale correctly represent the metric structure of temperature, it is nevertheless true that the presuppositions made when setting up these scales leave it open whether there is a question to be answered here at all.

As a matter of historical fact, different centigrade scales were set up using different substances (mercury, alcohol, ...). And the result of applying the function $|x-y| /|z-w|$ to the numbers the mercury scale assigned to some temperatures was not the same as the result of applying it to the numbers the alcohol scale assigned to the very same temperatures. So if temperature does have a metric structure at most one of these scales faithfully represents that structure. But without further theoretical input there is no way to decide which, if either, is faithful.

5. So far I have discussed the presuppositions made when setting up "empirical" temperature scales, scales that correlate temperature changes with properties (like volume changes) of particular substances (mercury, alcohol, air,...). Famously, William Thomson (later Lord Kelvin) proposed a scale for measuring temperature that did not make reference to the properties of any particular substance. (That is what is meant by the claim that his scale is an absolute temperature scale.) So what about Kelvin's scale? Does that scale presuppose that temperature had a metric structure?

Here is a brief description of the way the Kelvin scale is established. It is based on a theorem in thermodynamics. Consider heat engines that work in a cycle between two heat reservoirs at different temperatures: they absorb some amount

\footnotetext{
${ }^{6}$ Both of these quotations appear in Ernst Mach's "Critique of the Concept of Temperature," originally published 1896. An English translation appears as Appendix I of (Ellis 1966). The quotations appear on page 190 of that version. Chang (2004, 60-62) has more on early scientists' worries about the adequacy of their scales for measuring temperature.
} 
of energy $Q_{h}$ from a hot reservoir, do work, expel some amount of energy $Q_{c}$ to a cold reservoir, and return to their initial state. The values of $Q_{h}$ and $Q_{c}$ are different for different heat engines; they depend on how efficient the engine is. But Sadi Carnot proved that among all heat engines working between two heat reservoirs, reversible heat engines are maximally efficient. Thomson decreed, in effect, that the numbers assigned to temperature values on the Kelvin scale shall have the following property: if $T_{1}$ and $T_{2}$ are the numbers assigned by the scale to the temperatures of two heat reservoirs (and $T_{1}$ is assigned to the hotter reservoir), then

$$
\frac{T_{1}}{T_{2}}=\frac{Q_{1}}{Q_{2}}
$$

where $Q_{1}$ is the energy absorbed from the hotter reservoir in one cycle by a reversible heat engine (that works between the two reservoirs), and $Q_{2}$ is the amount of energy expelled to the colder reservoir in one cycle.

This constraint on the ratios of numbers assigned to temperatures is not yet enough to establish a temperature scale. But all that remains is to decree (arbitrarily) what number shall be assigned to one particular temperature. By convention 273.16 is assigned to water at its triple point.

Although Kelvin's scale is "absolute" the stipulations that establish it do not presuppose that temperature has a metric structure. (It certainly does not explicitly presuppose this. It does, of course, presuppose Carnot's theorem, and one might wonder whether Carnot's theorem presupposed this. It does not either; I will return to this in the next section.) Instead of presupposing that there are scale-independent facts about temperature ratios, the Kelvin scale is set up by first assigning ratios to numbers representing temperatures using ratios of energies. All this scale presupposes about the metric structure of any quantity, then, is that there are scaleindependent facts about ratios of energies.

None of the temperature scales we have looked at presuppose that temperature has a metric structure. And there is no more recent temperature scale that might have different presuppositions; the Kelvin scale is still the scale sanctioned by the International Bureau of Weights and Measures. But even though the Kelvin scale does not presuppose that temperature has a metric structure, one might think that 
the Kelvin scale's special properties do provide reason to think that temperature has a metric structure. I will return to this thought below.

Before proceeding with my argument I want to say something about why scientists who believed that temperature had a metric structure set up scales for measuring temperature that did not presuppose this.

A temperature scale is just an assignment of numbers to temperatures, and a faithful scale is one that faithfully represents the structure of temperature. There is nothing in the definition of "scale for measuring a quantity" that entails that it is within our power to use that scale to measure the value anything has for that quantity. It is possible, therefore, to set up a scale for measuring temperature that is completely impractical: a scale which is such that it is impossible for anyone to learn what temperature anything has on that scale.

It is not hard to see how to set up an impractical scale for measuring some quantity. We could set up an impractical length scale by choosing as our standard for length something to which we have no access-say, the height of the first human to set foot on Mars. Supposing humans do colonize Mars (and there is a single first human who gets there), each thing is assigned a length on this scale. But none of us can know how tall he is on this scale.

No temperature scale presupposes that temperature has a metric structure simply because (in the absence of theoretical advances) we could never determine what number any temperature value is assigned on such a scale. Even if we firmly believed that temperature has a metric structure, it is completely impractical to set up a temperature scale that by saying that the number that measures something's temperature on that scale is equal to $273.16 \frac{v}{W}$, where $v$ it that thing's value for temperature and $W$ is water's value for temperature at its triple point. The problem is that temperature is not a quantity we can directly measure. The scales we do use do not suffer from this problem. On them we can measure temperature indirectly, by finding the value for some other quantity (the volume of a gas for example) that we can measure directly.

6. It is time to turn to the main part of my argument. If there were reasons to think (before the advent of statistical mechanics) that temperature has a metric structure, 
those reasons are to be found in the theories that existed prior to the development of statistical mechanics in which temperature plays a role. What do those theories tell us? After briefly discussing the caloric theory of heat I will turn to thermodynamics.

Before we can know what a theory says about the structure of temperature we need to know where in a theory to look for this kind of information. And there are several ways in which a theory can indicate something about the structure of temperature.

One way a theory can do this is by explicitly saying what temperature is. The caloric theory is an example of an early (and false) theory that does this. Actually, there were several different caloric theories; but for our purposes it is enough to work with a simplified version of one of them. Now if one body is hotter than another then "heat will flow" from the first body to the second when they are put into thermal contact. The caloric theory explains this phenomenon by postulating the existence of a fluid — caloric - and identifying the temperature of a body with the density of the caloric fluid in that body. (Higher temperatures just are higher densities of caloric.) Caloric flows from regions of high caloric density to regions of low caloric density; so this theory has temperature behaving the way it should.

If the caloric theory is true then temperature has a metric structure. For the density of caloric in a body is just the amount of caloric divided by the volume of the body, and both volume and (presumably) amounts of caloric are quantities with metric structures.

But the caloric theory is false. So it is no guide to the structure of temperature. The theory we should be looking at, of course, is thermodynamics. Like the caloric theory, thermodynamics is false. But statistical mechanics assigns the false statements of thermodynamics extremely high probabilities. So it is close enough to being true to take what the theory says about temperature as good evidence about the structure of temperature.

But (unlike caloric theories) thermodynamics does not explicitly tell us what

\footnotetext{
${ }^{7}$ See (Chang 2004, 170-2) for brief descriptions of the dominant caloric theories. The simplified theory I present is closest to the Irvingist's theory; the main difference is that the Irvingists define a body's temperature as the total caloric contained in the body divided by the body's heat capacity, not by its volume.
} 
temperature is. It does not identify temperature with a combination of other quantities. Instead, thermodynamics tells us how temperature behaves-it formulates laws involving it. (Maybe by doing this it implicitly tells us what temperature is: it is whatever quantity plays the right role in its laws. But that is no help, since it was not until the development of statistical mechanics that we knew which quantity that was and could check directly whether it has a metric structure.) Does thermodynamics contain any information about the structure of temperature? It does, but the information is harder to extract. We need to look at what the laws of thermodynamics presuppose about the structure of temperature.

7. Even if thermodynamics does not tell us what temperature is, it can still be a guide to the structure of temperature. Consider the case of electromagnetism. Electromagnetism does not say anything helpful about what electric charge is. It does not identify electric charge with a combination of other, more fundamental quantities (the way, for example, it identifies electric current with the amount of charge that passes per unit time). But we can still see that electric charge has a metric structure by looking at the laws of electromagnetism. Those laws presuppose that electric charge has a metric structure. So if the laws of thermodynamics presuppose that temperature has a metric structure, then (since thermodynamics is approximately true) temperature does in fact have a metric structure.

There are (at least) two kinds of "laws" of thermodynamics in which temperature appears. It appears in the laws of thermodynamics properly so-called. And it also appears in equations of state like the ideal gas law.

I am going to discuss what the laws of thermodynamics properly so-called presuppose about the structure of temperature first. I am going to proceed this way because I do not see how the ideal gas law (for example) could indicate anything about the structure of temperature than the laws of thermodynamics do not. I will return to equations of state later.

Temperature appears in the zeroth, second, and third laws of thermodynamics. The zeroth law of thermodynamics ensures that it is consistent to say that there is such a quantity as temperature. That there is such a quantity as temperature entails that if $a$ and $b$ have the same temperature, and so do $b$ and $c$, then $a$ and $c$ also 
have the same temperature. But two bodies have the same temperature if and only if they are in thermal equilibrium. These two claims are consistent only if thermal equilibrium is an equivalence relation. And that is what the zeroth law says. But the zeroth law does not say anything more about the structure of temperature.

The third law of thermodynamics says that no thermodynamic system (or part of a thermodynamic system) may be cooled to absolute zero (in a finite number of operations). The third law, therefore, says that the (physically realizable) temperatures are bounded from below. This law places restrictions on the order type of the temperature values. But it does not presuppose that the temperature properties have a metric structure.

So what about the second law of thermodynamics? It is more complicated to say what the second law of thermodynamics presupposes about the structure of temperature, because there are many statements of the second law. I will start by looking at two of the most famous statements of the second law: Kelvin's statement and Clausius's statement. Their statements may be paraphrased as follows: 8

(K) No cyclical process is possible whose sole results are the transfer of energy by heating out of some heat reservoir and the performance of work.

(C) No cyclical process is possible whose sole result is the transfer of energy by heating out of a colder body and the transfer of energy by heating into a hotter body.

It is clear that neither of these statements presuppose that temperature has a metric structure. Kelvin's statement does not mention temperature differences at all. Clausius's statement does, but it presupposes only that bodies are ordered from lower to higher temperatures. The magnitudes of temperature differences play no role in it.

8. The most well-known statement of the second law of thermodynamics uses the concept of entropy:

(E) For any process undergone by an adiabatically isolated system that begins

${ }^{8}$ Uffink (2001) has a comprehensive discussion of the many versions of the second law. 
and ends in an equilibrium state, the entropy of the final state is not less than the entropy of the initial state.

(A system is adiabatically isolated if energy cannot enter or leave it through heating; energy is permitted to enter or leave only through the performance of work.) The word "temperature" does not even appear in (E) so it is not immediately obvious what, if anything, (E) presupposes about the structure of temperature. But (E) does employ the concept of entropy, which (in classical thermodynamics) is defined in terms of temperature, so (E) may indirectly presuppose something about the structure of temperature.

However, "presuppose" is not really the right word here. If (E) makes it reasonable to believe that temperature has a metric structure it is not because (E) presupposes that temperature has that structure. It is for a different reason. And, in fact, figuring out what $(\mathrm{E})$ indicates about the structure of temperature is not at all easy. Let me explain why.

In standard presentations of classical thermodynamics $(\mathrm{E})$ is not the fundamental statement of the second law. Instead, some other statement of the second law that does not presuppose that temperature has a metric structure (like the Kelvin or Clausius statements, or some generalization of them, like the one Serrin [1979] uses) is used to prove that there is such a state function as entropy. Then it is proved further that $(\mathrm{E})$ is equivalent to those statements..$^{9}$ Kelvin's temperature scale plays an important role in this proof.

I want to look at some of the details of this proof. But we are already in a position to say a few things about what (E) might indicate about the structure of temperature. There is some reason to think that $(\mathrm{E})$ does not indicate that temperature has a metric structure. For $(\mathrm{E})$ is derived from other statements of the second law that do not presuppose that temperature has a metric structure. How could (E) say, or presuppose, more about the structure of temperature than the principles from which it is derived? If it did it would seem like something had come from nothing.

On the other hand, there is also reason to think that $(\mathrm{E})$ does indicate that

\footnotetext{
${ }^{9}$ Technically, (C) and (K) are not equivalent, so are not both equivalent to (E). But it took the recognition of the existence of negative absolute temperatures to see this (see Uffink 2001, 325-30).
} 
temperature has a metric structure. For the Kelvin scale plays an important role in the proof that entropy is a state function. Now suppose that the only scales that can play this role agree with Kelvin's on the ratios of numbers assigned to temperature values. Then this class of scales is the unique class that plays an important and foundational role in the theory. Maybe that makes it reasonable to think that there are scale-independent facts about the ratios of temperature values. For if there are such facts then we can explain why this class, and not some other class, plays this important role. It plays this role because it is the class of scales that faithfully represent the ratio structure of temperature.

That is why it is not clear what we should think about the structure of temperature in the light of (E). To try to form a more settled opinion let us look more closely at some of the details of the proof that entropy is a state function.

The entropy difference between two equilibrium states $s_{1}$ and $s_{2}$ is defined to be

$$
\Delta S=\int_{s_{1}}^{s_{2}} \frac{d Q}{T},
$$

where the integral is taken along any "reversible" path through the space of equilibrium states that connects $s_{1}$ and $s_{2}$. Here $d Q$ is the one-form giving the energy absorbed by heating during an infinitesimal change and $T$ is the system's temperature on the Kelvin scale (for typographical reasons I write " $d Q$ " without the bar on the " $d$ "; $d Q$ is not an exact differential).

This definition of entropy makes sense only if we get the same result no matter what path from $s_{1}$ to $s_{2}$ we choose. The proof that this is so usually proceeds, in outline, as follows. First the second law (Clausius version) is used to prove Carnot's Theorem (mentioned above):

- No heat engine operating between two heat reservoirs is more efficient than a reversible engine.

Now the efficiency of a heat engine operating between two heat reservoirs is equal to $1-Q_{c} / Q_{h}$. So Carnot's theorem says that for any heat engine,

$$
1-\frac{Q_{c}}{Q_{h}} \leq 1-\frac{Q_{r c}}{Q_{r h}},
$$


where $Q_{r c}$ and $Q_{r h}$ are the energies absorbed and emitted by a reversible engine. That is,

$$
\frac{Q_{r c}}{Q_{r h}} \leq \frac{Q_{c}}{Q_{h}} .
$$

If we use Kelvin's scale to measure temperature, then by definition

$$
\frac{Q_{r c}}{Q_{r h}}=\frac{T_{c}}{T_{h}} .
$$

Equations (1) and (2) entail

$$
\frac{Q_{h}}{T_{h}} \leq \frac{Q_{c}}{T_{c}},
$$

or, replacing $Q_{c}$ with $-Q_{c}$ so that $Q_{c}$ denotes the amount of energy that the engine absorbs from the cold reservoir,

$$
\sum \frac{Q}{T} \leq 0
$$

The next step (the details of which I omit) is to argue that, in general, for any cyclic process (not just for heat engines),

$$
\oint \frac{d Q}{T} \leq 0
$$

Now for any reversible cyclic process, both $\oint d Q / T \leq 0$ and $\oint d Q / T \geq 0$. This (together with the claim that any two equilibrium states can be connected by a reversible process) implies that $\oint d Q / T=0$, and this implies that $\int_{s_{1}}^{s_{2}} d Q / T$ is independent of path-entropy is a state function.

What is important about this proof for our purposes is the following. Only if $T$ stands for the numbers assigned to temperatures on the Kelvin scale or a scale similar to the Kelvin scale (a scale obtained from it by multiplying all numbers it assigns to temperature values by some constant) is $\int_{s_{1}}^{s_{2}} d Q / T$ independent of path.

Now I earlier presented an argument that this proof provides a reason to believe that temperature has a metric scale. That main premise at work in that argument is the following conditional:

(*) If scales of temperature similar to the Kelvin scale are the only scales that 
can be used to prove that entropy is a state function, then temperature has a metric structure that these scales faithfully represent.

This is a plausible claim. However, I do not think its antecedent is true.

Consider the temperature scale that assigns to any temperature value the number $-1 / T$, where $T$ is the number assigned to that temperature value on the Kelvin scale. This scale agrees with the Kelvin scale on how temperature values are ordered, but not on their ratios, or on the ratios of temperature intervals. ${ }^{10}$ I will use " $\tau$ " to stand for the numbers assigned to temperatures on this new scale. This scale can also be used to prove that entropy is a state function. For the integral $-\int_{s_{1}}^{s_{2}} \tau d Q$ is independent of path, and is equal to the entropy difference between $s_{1}$ and $s_{2}$. As far as being of use to prove that entropy is a state function, then, the Kelvin scale and the $\tau$ scale work equally well. And that means we cannot appeal to (*) to justify belief that temperature has a metric structure.

Of course if $f(x)$ is any smoothly increasing (and so invertible) function from numbers to numbers we can define a new temperature scale that assigns the number $f(T)$ to the value to which the Kelvin scale assigns the number $T$. If we let " $\bar{T}$ " denote numerical temperature values on this scale, then the integral $\int_{s_{1}}^{s_{2}} d Q / f^{-1}(\bar{T})$ will be independent of path. So for each such function $f$ we can define a temperature scale that is a counterexample to the antecedent of $\left(^{*}\right)$. But if $f$ is some extremely complicated function then this might look like a trick. We apply $f$ to define the new scale, but then apply its inverse in the definition of entropy; it still looks like the Kelvin scale is doing the real work.

But I do not think that the argument that appeals to the $\tau$ scale is a trick. The situation we are in with respect to $\tau$ is this: there is a temperature scale (the Kelvin scale) with the property that when you divide $d Q$ by temperature on this scale you get a differential whose integral is independent of path (and we use this integral to define entropy); and there is another scale (the $\tau$ scale) with the property that when you multiply $d Q$ by temperature on this scale you get a differential whose integral

\footnotetext{
${ }^{10}$ It also assigns all temperature values negative numbers. (Actually, it assigns negative numbers to values to which the Kelvin scale assigns positive numbers. But I am ignoring the existence of temperature values to which the Kelvin scale itself assigns negative numbers).
} 
is independent of path (and we use this integral to define entropy). When you put it that way, it does not look like the proof that uses the $\tau$ scale must be thought of as parasitic on the proof that uses the Kelvin scale, or that it is the Kelvin scale that is doing the real work. Multiplication is no more complicated, and no less natural, than division.

So there are temperature scales that are equally useful when it comes to proving that there is such a state function as entropy, and those scales do not agree on the ratios of numbers assigned to temperature values. I think this shows that looking at (E) and the proof that entropy is a state function does not give us grounds for believing that temperature has a metric structure.

9. Temperature appears in thermodynamics in places other than the laws of thermodynamics. It also appears in equations of state, like the ideal gas law. Now I said earlier that I do not see how the ideal gas law could indicate that temperature has a metric structure if the fundamental laws of thermodynamics do not. Still, it is worth looking in to how we should think about equations like the ideal gas law if we accept that the fundamental laws of thermodynamics do not indicate that temperature has a metric structure.

One might use the ideal gas law to argue that temperature has a metric structure (and also establish that this structure is correctly represented by air thermometers and not by mercury or alcohol thermometers) as follows. The ideal gas law is $P V=N k T$. Thus when the pressure of a sample of gas is held constant its temperature is proportional to its volume. (This special case of the ideal gas law is Charles' law.) So if a sample of gas is heated (at constant pressure) until its volume doubles, then its temperature has also doubled. But to say that the temperature has doubled is to say that the scale-independent ratio of the final temperature to the initial temperature is two. Doesn't this settle that there are scale-independent temperature ratios?

Well, does it follow from $P V=N k T$ that doubling a gas's volume (at constant pressure) doubles its temperature? Let's think about what $P V=N k T$ means (and more generally what equations between physical quantities mean). It does not mean that the result of multiplying a gas's value for pressure and its value for volume is 
identical to the result of multiplying its value for temperature and $N k$. You can only multiply numbers, and pressure values, volume values, and temperature values are not numbers. They are properties that are assigned numbers relative to scales of measurement. What $P V=N k T$ means, then, is that the result of multiplying the number that is assigned to the gas's pressure value (on a certain scale) and the number that is assigned to the gas's volume value (on a certain scale) is identical to the result of multiplying the number assigned to its temperature value on the Kelvin scale and $N k ! \sqrt{11}$

So it does not follow from $P V=N k T$ alone that doubling a gas's volume doubles its temperature. This only follows if we also assume that temperature has a metric structure that is correctly represented by the Kelvin scale (since it is relative to that scale, and scales similar to it, that $P V=N k T$ is correct). But this assumption is just what is in question. So this argument that temperature has a metric structure is circular.

There is, however, more to be said. The questions I have been asking about the structure of temperature can (and have) been asked about other quantities. There are analogous debates about what laws involving those quantities indicate about the structure of those quantities. One famous example is duration: do durations have a metric structure $\left[{ }^{12}\right.$ Look at $F=m a$. There is an argument analogous to the one I just gave that starts from $F=m a$ and concludes that accelerations have a metric structure (and if they do, so do durations, in terms of which they are defined). And there is an analogous reply: $F=m a$ is true relative to one scale for measuring duration (or, better, one coordinate system for time). If we wanted to use a different coordinate system, we would have to use a different equation to express this law. Maybe duration does have a metric structure (that is faithfully represented by one of these coordinate systems), but that it is a law that $F=m a$ does not give us reason

\footnotetext{
${ }^{11}$ Usually we do not want to write equations in physics so that they are only true relative to a particular scale for measuring a quantity. Instead write the equations so that they are true relative to a whole family of similar scales (scales that agree on the ratios of numbers assigned to quantity values). Equations like that are "dimensionally homogenous."

${ }^{12}$ Newton (2004) famously argued that they do; Barbour (1999) is a recent proponent of the view that they do not.
} 
to think so.

In the case of duration, however, this is not the end of the story. To settle the issue we write Newton's theory (including his second law) in a way that is valid in any coordinate system whatever. This is the "generally covariant" formulation of the theory (Friedman [1983, 71-124] has the details.) When we do that the temporal metric appears explicitly in the equations expressing the theory. This is conclusive evidence that in Newtonian mechanics duration has a metric structure.

One might hope that something similar can be done for the case of temperature. Write the ideal gas law (and other equations of thermodynamics) so that it is true no matter what scale we choose to measure $P, V$, and $T$. Then look to see if metric structure for $T$ appears explicitly in that formulation of the theory. But I know of no formulation of thermodynamics like this. So we cannot use this strategy to settle the question.

The best we can do, I think, is appeal to simplicity. The equations for thermodynamics take on a relatively simple form when written relative to the Kelvin scale. Perhaps this gives us some reason to think temperature has a metric structure that is faithfully represented by the Kelvin scale. But these reasons are weak. (For one thing, it is not obvious that these equations are less simple when written relative to the $\tau$ scale.)

10. When we turn to statistical mechanics there is a clear and straightforward case to be made for the proposition that temperature has a metric structure. I will close by rehearsing it.

Statistical mechanics, unlike thermodynamics but like the caloric theory, explicitly says what temperature is. In statistical mechanics temperature is not treated as a fundamental quantity and is identified with a combination of more fundamental quantities.

Statistical mechanics says that a body's temperature is the derivative of its internal energy with respect to its entropy (with external parameters held fixed) 13

\footnotetext{
${ }^{13}$ It does not say that a body's temperature is the average kinetic energy of its constituent molecules. This claim is false for many reasons; for one thing, there are thermodynamic systems (ideal paramagnets, for example) that can have negative
} 
In symbols this is

$$
\frac{1}{T}=\frac{\partial S}{\partial E}
$$

Of course, we can infer nothing from (3) about the structure of temperature unless we know something about the structure of entropy. But statistical mechanics also tells us what entropy is: the (Boltzmann) entropy of a system is the logarithm of the number of possible microstates (or: the measure of the set of possible microstates) of that system that are compatible with the system's macrostate, times Boltzmann's constant. ${ }^{14}$ This quantity clearly has a metric structure. (The scale-independent ratio of system A's entropy to system B's is $r$ iff A has $n^{r}$ available microstates, where $n$ is the number of microstates available to B.) Energy also has a metric structure. Since energy and entropy are quantities with metric structures, it follows from (3) that temperature is as well. 15

\section{References}

Baierlein, Ralph. 1999. Thermal Physics. New York: Cambridge University Press. Barbour, Julian. 1999. The End of Time. New York: Oxford University Press.

Bureau International des Poids et Mesures. 2006. The International System of Units. 8th edition. English version. Available online at http://www.bipm.org/utils/common/pdf/si_brochure_8_en.pdf.

Chankg, Hasok. 2004. Inventing Temperature: Measurement and Scientific Progress. New York: Oxford University Press.

Ellis, Brian. 1966. Basic Concepts of Measurement. New York: Cambridge University Press.

Friedman, Michael. 1983. Foundations of Space-Time Theories. Princeton: Princeton University Press.

Krantz, David, R. Duncan Luce, and Amos Tversky. 1971. Foundations of Mea-

temperatures (on the Kelvin scale); but kinetic energy cannot be negative. (This misconception is dispelled in standard thermal physics textbooks. See, for example, [Baierlein 1999, 347-49] or [Schroeder 2000, 85-91].)

${ }^{14}$ Another tradition, tracing back to Gibbs' work, defines entropy differently. Uffink (2007) provides a good discussion of what Gibbs says about entropy.

${ }^{15}$ Thanks to an anonymous referee for helpful comments. 
surement Volume 1: Additive and Polynomial Representations. New York: Academic Press.

Mundy, Brent. 1987. “The Metaphysics of Quantity.” Philosophical Studies 51:2954.

Newton, Isaac. 2004. "The Principia [1687, first edition]." In Newton: Philosophical Writings, ed. Andrew Janiak, 40-93. New York: Cambridge University Press.

Poincaré, Henri. 1905. Science and Hypothesis. New York: The Science Press.

Reichenbach, Hans. 1957. The Philosophy of Space and Time. New York: Dover Publications.

Schroeder, Daniel. 2000. An Introduction to Thermal Physics. New York: Addison Wesley Longman.

Serrin, James. 1979. "Conceptual Analysis of the Classical Second Law of Thermodynamics." Archive for Rational Mechanics and Analysis 70:355-71.

Stevens, S. S. 1946. "On the Theory of Scales of Measurement." Science 103:67780.

Uffink, Jos. 2001. "Bluff Your Way in the Second Law of Thermodynamics." Studies in History and Philosophy of Modern Physics 32:305-94.

_ _ - "Compendium of the Foundations of Classical Statistical Physics." In Handbook for the Philosophy of Physics, ed. Jeremy Butterfield and John Earman, 924-1074. Amsterdam: Elsevier. 